\title{
Immunohistochemical Study on Perinatal Development of Rat Superoxide Dismutases in Lungs and Kidneys
}

\author{
KOHTARO ASAYAMA, HIDEMASA HAYASHIBE, KAZUSHIGE DOBASHI, NORIHIKO UCHIDA, \\ MAKIO KOBAYASHI, AKIRA KAWAOI, AND KIYOHIKO KATO \\ Department of Pediatrices [K.A., H.H., K.D., N.U., K.K.] and Pathology [A.K.], Yamanashi Medical College \\ Yamanashi 409-38, Japan and Department of Pathology, Tokyo Woman's Medical College, \\ Tokyo 162, Japan [M.K.]
}

\begin{abstract}
It has been reported that levels of antioxidant enzymes are low in fetal rat lungs and kidneys, and that they increase rapidly during late gestation. Among the antioxidant enzymes, both copper-zinc and manganese superoxide dismutases (CuZnSOD and MnSOD) are assumed to play a key role in protection against oxidative tissue injury. To determine the nature of the rapid perinatal increase in CuZnSOD and MnSOD, immunoenzyme staining was performed in the lungs and kidneys of fetal (d 18 and 20 of gestation) and neonatal (d 22) rats. The CuZnSOD and MnSOD in the homogenates were assayed by RIA, and they were found to be higher in the neonatal organs than in the respective fetal organs. The neonatal bronchiolar epithelium was stained for both CuZnSOD and MnSOD more intensely than the fetal one. The $\mathrm{Cu}$ ZnSOD staining in the neonatal alveolar wall was more intense than that in the fetal one. There was a significant reactivity for MnSOD in the neonatal, but not in the fetal, alveolar walls. In the kidneys, the reactivities for $\mathrm{Cu}$ $\mathrm{ZnSOD}$ and MnSOD were confined to the undifferentiated tubules. Although the tubules were increased in numbers in the neonatal kidneys, the intensity of the staining for both CuZnSOD and MnSOD was unchanged. The histochemical study disclosed that CuZnSOD and MnSOD increased in the kidneys in a manner different from that in the lungs. The low concentration of both $\mathrm{CuZnSOD}$ and MnSOD in the fetal lung tissues may contribute to the vulnerability to oxygen toxicity. Such changes in the concentrations in specific tissues were not delineated in the kidneys. (Pediatr Res 29: 487-491, 1991)
\end{abstract}

\section{Abbreviations}

CuZnSOD, copper-zinc superoxide dismutase MnSOD, manganese superoxide dismutase SOD, superoxide dismutase

In recent years, cell injury mediated by reactive oxygen species has drawn increasing attention from neonatologists. Abrupt increase of tissue oxygen concentration after delivery may induce oxidative injury to vulnerable tissues. There is evidence suggesting that fetal lungs have a low-capacity antioxidant defense

Received May 29, 1990; accepted December 17, 1990

Correspondence and reprint requests: Kohtaro Asayama, M.D., Department of Pediatrics, Yamanashi Medical College, 1110 Shimokato, Tamahocho. Nakakomagun, Yamanashi 409-38, Japan.

Supported in part by Grant-in-Aid 63570431 from the Ministry of Education, Science and Culture of Japan. system (1-4), and this is postulated to contribute to the development of bronchopulmonary dysplasia in preterm neonates. Superoxide anion radical, which is generated by one electron reduction of molecular oxygen, is considered to be an initiator of free radical chain reactions leading to oxidative cell injury in certain experimental models (5). Thus, SOD (EC 1.15.1.1), a scavenger of superoxide, may play a key role in the protection against free radical injury. Intracellular SOD is found in two forms: CuZnSOD and MnSOD. Using specific RIA (6) for both rat CuZnSOD and MnSOD, we have demonstrated that both SOD increase markedly during the late gestational period (i.e. from d 18 to 22 of gestation) in fetal rat lungs and kidneys (7).

The immunoenzyme staining for rat CuZnSOD and MnSOD, which has been developed by us recently (8), revealed that there was a wide range of variability in the expression of both SOD from cell to cell in adult rat tissues. To our knowledge, immunohistochemical localization of CuZnSOD and MnSOD in fetal and neonatal rat tissues has not been reported previously. To further determine the nature of the rapid perinatal increase in SOD, lungs and kidneys of both fetus and neonate were stained for CuZnSOD and MnSOD, and the immunoreactive SOD in these organs were also measured. Our present study revealed that the increase in SOD occurred in certain specific tissues of these rapidly growing organs. Different characteristics of the increase between lungs and kidneys were also shown.

\section{MATERIALS AND METHODS}

Animal treatment. Breeding was accomplished by placing male and female Sprague-Dawley rats (Japan SLC Inc., Shizuoka, Japan) together overnight. The midpoint of cohabitation period was considered as the onset of pregnancy. Premature pups were delivered by hysterotomy with the dam under pentobarbital anesthesia $(201 \mu \mathrm{mol} / \mathrm{kg} ; 50 \mathrm{mg} / \mathrm{kg})$ on $\mathrm{d} 18$ and 20 of gestation, and decapitated immediately after the delivery. Neonates were killed within $24 \mathrm{~h}$ after delivery under light ether anesthesia. The lungs and kidneys were excised, washed with PBS, and blotted with gauze.

$R I A$ for SOD. The organs were weighed and pooled to obtain at least $80 \mathrm{mg}$ of tissue for each sample, and then kept frozen at $-80^{\circ} \mathrm{C}$. The tissues were homogenized with 20 times their volume of $10 \mathrm{mM}$ potassium phosphate buffer containing $81 \mu \mathrm{M}$ digitonin ( $\mathrm{pH} 7.4$ ), and then sonicated on ice for $30 \mathrm{~s}$. The sonicate was centrifuged at $13000 \times g$ for $5 \mathrm{~min}$, and the supernatant was obtained. This served as the sample for RIA. The methods of RIA for rat CuZnSOD and MnSOD have been described (6). The data were expressed as means \pm SEM. The statistical significance was determined by the method of least significant difference calculated after one-way analysis of variance.

Immunoenzyme staining for SOD. The specificity of each 
Table 1. Immunoreactive SOD in homogenate of lung and kidney*

\begin{tabular}{|c|c|c|c|c|c|c|}
\hline & \multicolumn{3}{|c|}{ Lung } & \multicolumn{3}{|c|}{ Kidney } \\
\hline & $\begin{array}{c}18 \mathrm{~d} \text { fetus } \\
(n=9)\end{array}$ & $\begin{array}{c}20 \mathrm{~d} \text { fetus } \\
(n=9)\end{array}$ & $\begin{array}{l}\text { Neonate } \\
(n=8)\end{array}$ & $\begin{array}{c}18 \mathrm{~d} \text { fetus } \\
(n=4)\end{array}$ & $\begin{array}{c}20 \mathrm{~d} \text { fetus } \\
(n=9)\end{array}$ & $\begin{array}{l}\text { Neonate } \\
(n=8)\end{array}$ \\
\hline $\begin{array}{l}\text { CuZnSOD } \\
\text { MnSOD }\end{array}$ & $\begin{array}{c}1.41 \pm 0.06 \\
77 \pm 3\end{array}$ & $\begin{array}{c}1.99 \pm 0.05 \dagger \\
89 \pm 12\end{array}$ & $\begin{array}{l}2.90 \pm 0.12 \ddagger \\
218 \pm 16 \ddagger\end{array}$ & $\begin{array}{l}1.13 \pm 0.07 \\
154 \pm 15\end{array}$ & $\begin{array}{l}1.80 \pm 0.03 \dagger \\
364 \pm 9 \dagger\end{array}$ & $\begin{array}{c}2.51 \pm 0.08 \ddagger \\
503 \pm 14 \pm\end{array}$ \\
\hline
\end{tabular}

* Data are means \pm SEM. Values are $\mathrm{nmol} / \mathrm{g}$ wet tissue for $\mathrm{CuZnSOD}$ and $\mathrm{pmol} / \mathrm{g}$ wet tissue for MnSOD.

$\dagger p<0.001$ (vs $18 \mathrm{~d})$.

$\ddagger p<0.001$ (vs $20 \mathrm{~d}$ ).

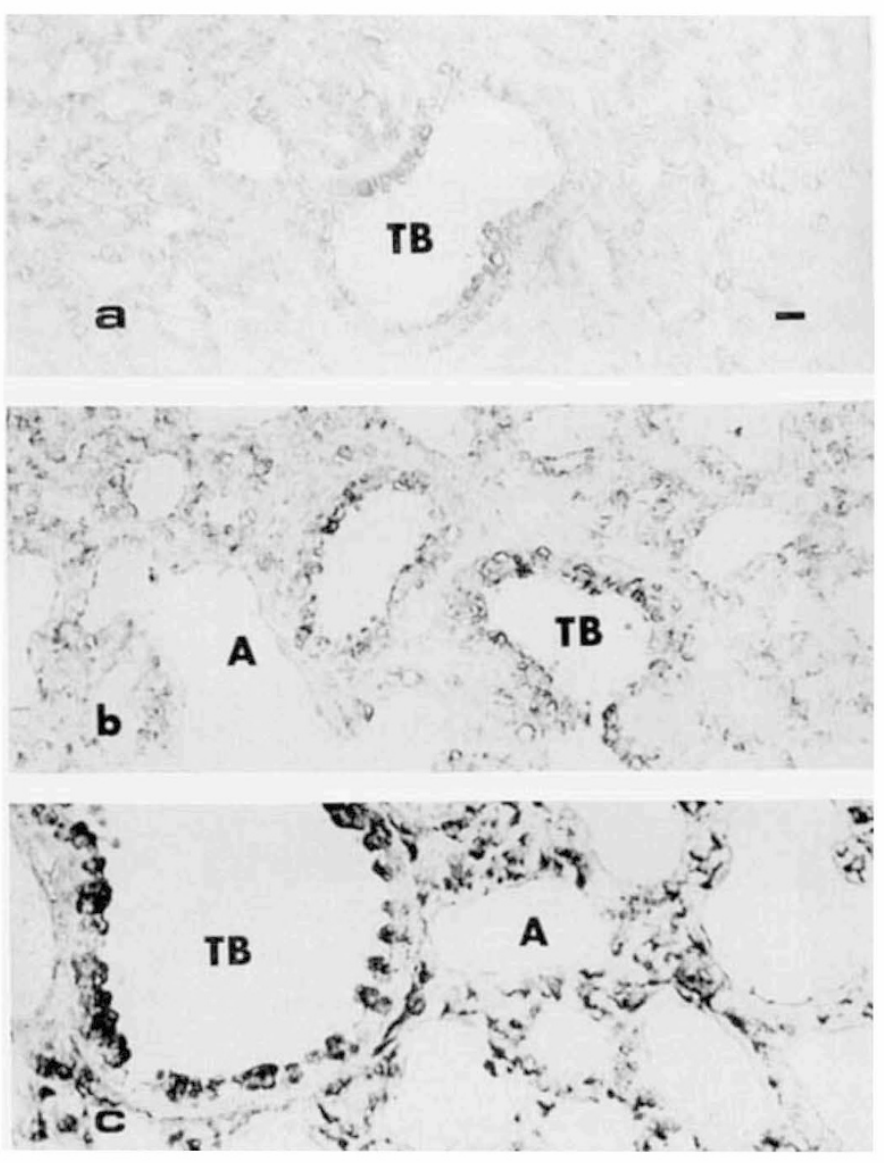

Fig. 1. Photomicrographs of fetal and neonatal rat lungs stained for CuZnSOD. $a$, Fetal lung (d 18); $b$, fetal lung (d 20); and $c$, neonatal lungs. The CuZnSOD reactivity is higher in the neonatal lungs than in the fetal lung. Original magnification $\times 200$. Bar indicates $10 \mu \mathrm{m}$. $A$, alveolus; TB, terminal bronchiole.

antiserum was established when the RIA was developed (6). The immunoenzyme staining for both CuZnSOD and MnSOD was performed as described previously (8). The tissues were fixed with $10 \%$ buffered formalin and embedded in paraffin. The specimens were cut into $4-\mu \mathrm{m}$ thick slices. The tissue sections were washed for $15 \mathrm{~min}$ with three changes of PBS between each step. Deparaffinized tissue sections were treated with $0.88 \mathrm{M}$ $\mathrm{H}_{2} \mathrm{O}_{2}$ for 10 min to inactivate endogenous peroxidase activity. Sections were then treated with $10 \%$ normal goat serum for 30 min to block nonspecific binding. The sections were incubated with the diluted antiserum (1:10000 for anti-CuZnSOD and 1:5000 for anti-MnSOD) overnight at $4^{\circ} \mathrm{C}$, then sequentially with $1 \%$ anti-rabbit IgG fraction antigen binding fraction labeled with horseradish peroxidase for $60 \mathrm{~min}$, and $0.56 \mathrm{mM}$ diaminobenzidine containing $1.47 \mathrm{mM} \mathrm{H}_{2} \mathrm{O}_{2}$ for $5 \mathrm{~min}$. Finally, the sections were mounted by Permount (Fisher Scientific Co., Pittsburgh, PA). As controls, tissue sections were treated in the same way,
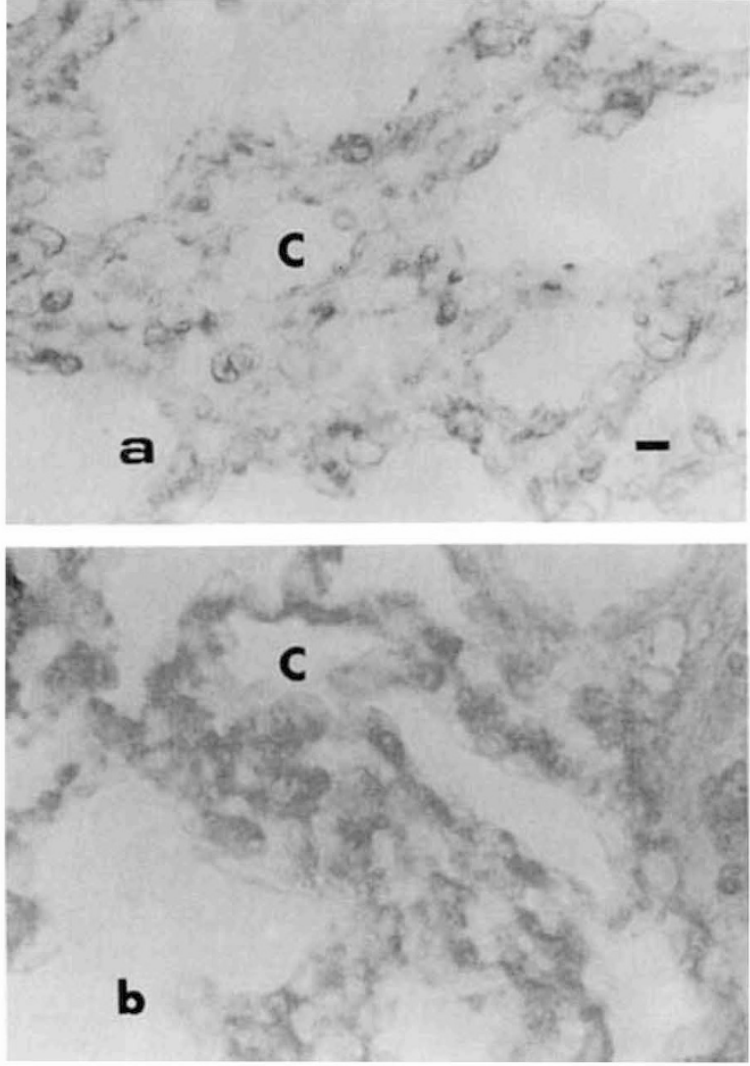

Fig. 2. CuZnSOD staining in lung at high magnification. $a$, Fetal lung (d 20); $b$, neonatal lung. The alveolar wall including capillary endothelium in the neonatal lung is stained more intensely than that in the fetal lung. Original magnification $\times 400 . B a r=5 \mu \mathrm{m}$. $C$, capillary endothelium.

except that antisera were replaced by normal rabbit serum at the same dilutions (8).

Morphometry. Morphometry was performed with an image analyzer (IBAS-2000; Zeiss, Munich, Germany). The sections were observed under a light microscope at a magnification of $200 \times$. Histologic images were transmitted to the image analyzer by a color TV camera (ITC-350M; Ikegami, Tokyo, Japan). The images of the staining profiles were projected on the screen by the specific gray threshold level (9). All images in three visual fields, which were randomly selected, were measured for each sample, and the area percentage was computed.

\section{RESULTS}

Immunoreactivity for SOD. Table 1 lists the immunoreactivity for both CuZnSOD and MnSOD in the homogenates of lungs and kidneys. The concentrations of both SOD in each organ were significantly higher in the $\mathrm{d} 20$ fetus than in the $\mathrm{d} 18$ fetus, and also higher in the neonates than in the d 20 fetus, although the difference in the lung MnSOD between d 18 and 20 was not significant. The CuZnSOD level in the lungs was comparable to 


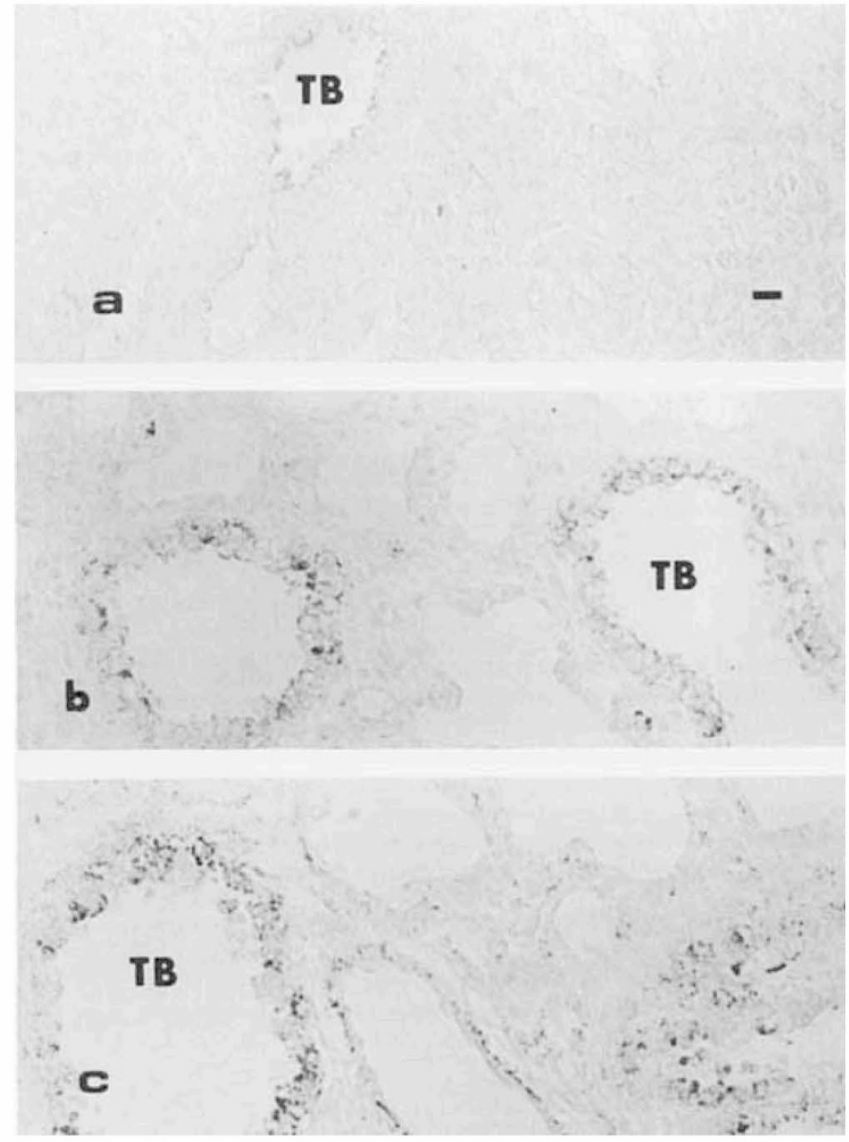

Fig. 3. Fetal and neonatal rat lungs stained for MnSOD. $a$, Fetal lung (d 18); $b$, fetal lung (d 20); and $c$, neonatal lung. In the fetal lungs, positive stainings are found only in the epithelium of the terminal bronchiole $(T B)$. Both terminal bronchiole and alveoli are stained in the neonatal lungs. Original magnification $\times 200$. Bar $=10 \mu \mathrm{m}$.

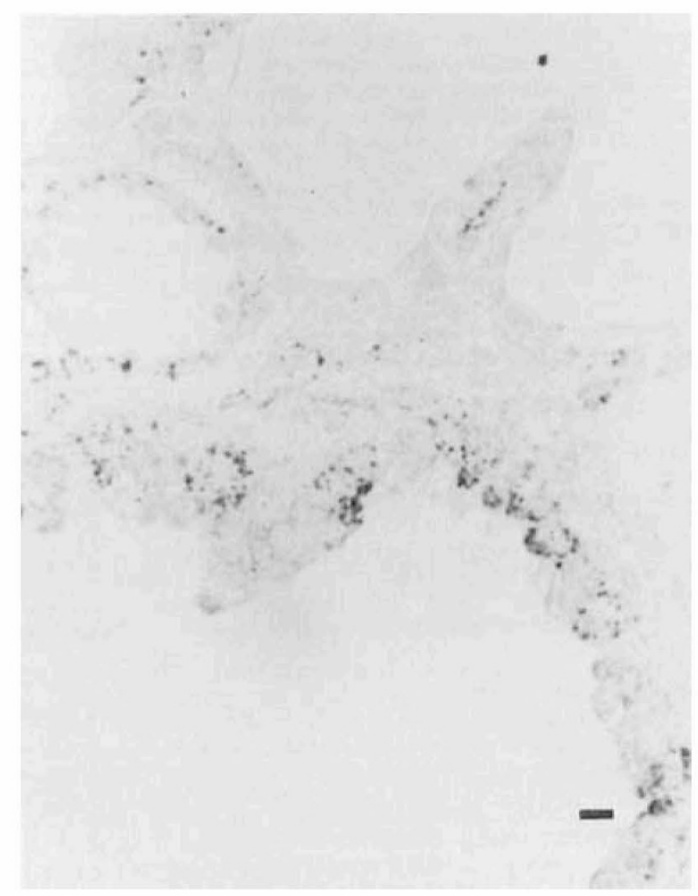

Fig. 4. MnSOD staining in neonatal lung at high magnification. 'ositive structures are seen both in bronchiole and alveolus. Original nagnification $\times 400$. Bar $=5 \mu \mathrm{m}$.
Table 2. Area percentage of staining for CUZnSOD and MnSOD in lung and kidney*

\begin{tabular}{cccccccc}
\hline & \multicolumn{3}{c}{ Lung } & \multicolumn{3}{c}{ Kidney } \\
\cline { 2 - 7 } \cline { 5 - 7 } CuZnSOD & 5.7 & 13.6 & 20.8 & 6.7 & 27.3 & 45.9 \\
& 5.5 & 14.5 & 24.2 & 7.9 & 27.9 & 38.5 \\
& 6.5 & d fetus & Neonate & 18 & d fetus & 20 d fetus Neonate \\
\hline \multirow{4}{*}{ MnSOD } & 8.2 & 11.3 & 24.5 & 6.2 & 28.6 & 38.2 \\
& 0.07 & 0.37 & 1.47 & 14.2 & 28.4 & 38.7 \\
& 0.05 & 0.32 & 1.20 & 13.4 & 31.7 & 32.7 \\
& 0.08 & 0.56 & 1.82 & 13.7 & 31.3 & 41.6 \\
\hline
\end{tabular}

* The image of the staining was measured, and the area percentage to visual field was computed.

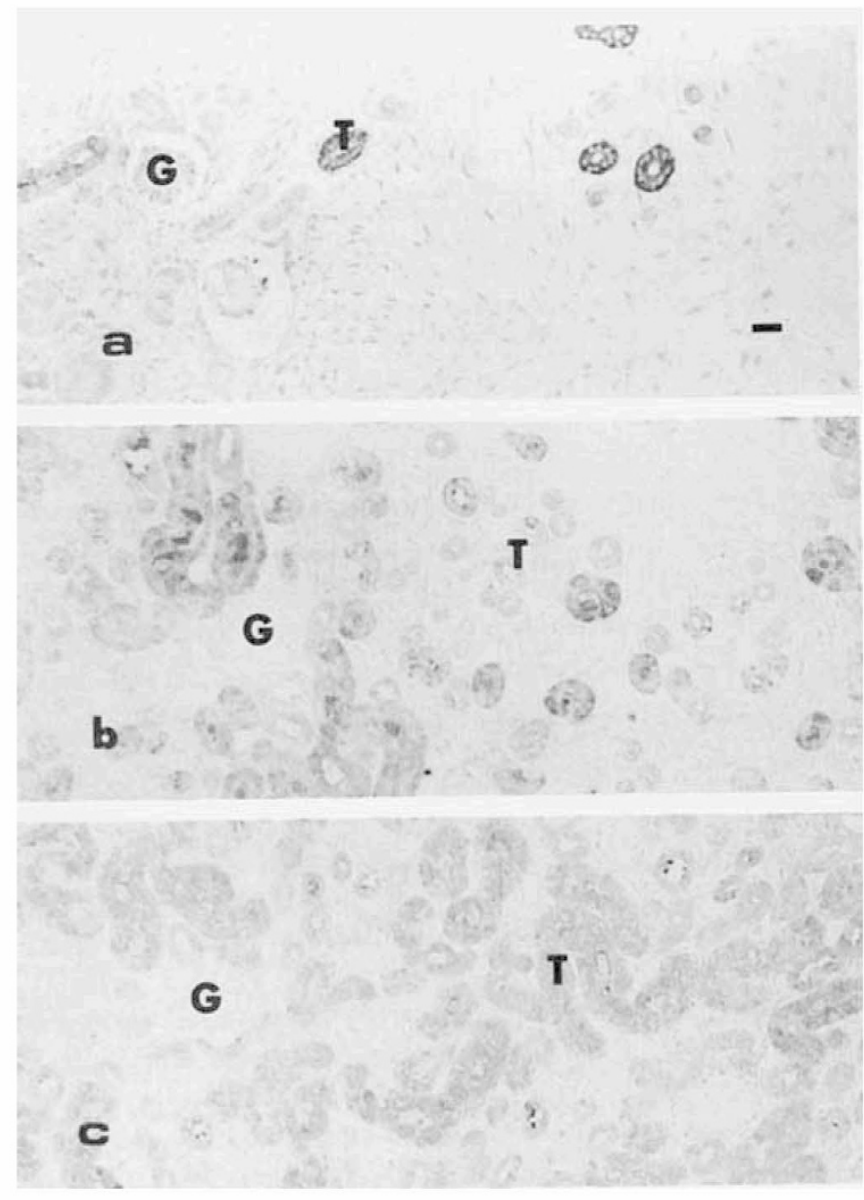

Fig. 5. Fetal and neonatal rat kidneys stained for CuZnSOD. $a$, Fetal kidney (d 18); $b$, fetal kidney (d 20); and $c$, neonatal kidney. Crosssectional view of juxtamedullary region. Only the renal tubules are stained. The section of the neonatal kidney contains more tubules than that of the fetal kidney. Original magnification $\times 100 . B a r=20 \mu \mathrm{m}$. $G$, glomerulus; $T$, tubulus.

that in the kidneys at each time point. The MnSOD represented $12 \%$ of the total SOD in the kidneys of the d 18 fetus, and it represented $17 \%$ of the total SOD in the kidneys of the $\mathrm{d} 20$ fetus and neonates. The relative concentration of MnSOD in the lungs was much lower than that in the kidneys. The MnSOD represented $4 \%$ of the total SOD in the d 20 fetal lungs and, even after a drastic increase, it still represented only $7 \%$ of the total SOD in the neonatal lungs.

Immunohistochemical localization of SOD. Positive stainings for $\mathrm{CuZnSOD}$ were found only in the bronchial epithelium of the $\mathrm{d} 18$ fetus, but were found in the bronchiolar epithelium and alveolar walls of both the d 20 fetus and neonates (Fig. 1). The staining intensity in the bronchiolar epithelium increased with age. Similarly, the CuZnSOD staining in the alveolar wall, in- 


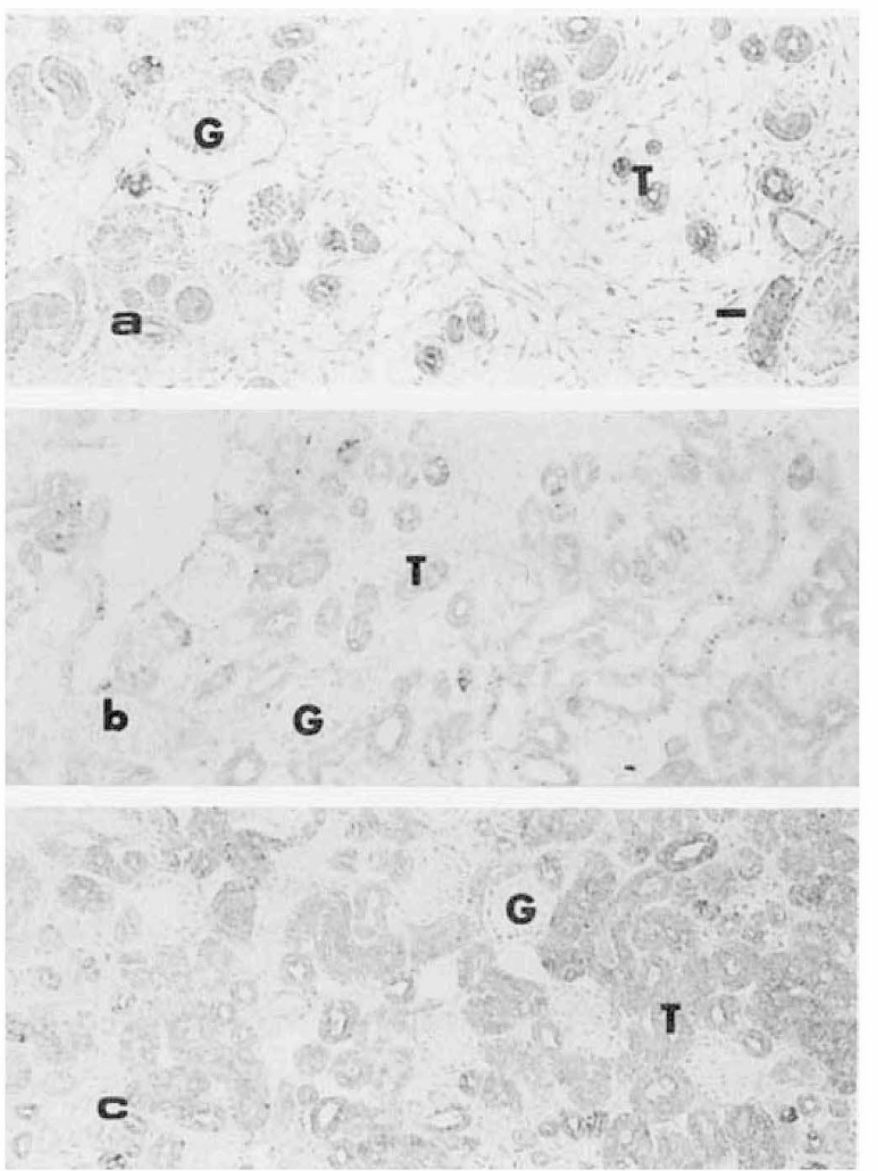

Fig. 6. Fetal and neonatal rat kidneys stained for MnSOD. $a$, Fetal kidney (d 18); $b$, fetal kidney (d 20); and $c$, neonatal kidney. The renal tubules in fetal (d 18 and 20) and neonatal kidneys are stained moderately. Original magnification $\times 100 . B a r=20 \mu \mathrm{m}$. $G$, glomerulus, $T$, tubulus.

cluding epithelia and capillary endothelia, also increased during this period (Fig. 2). The MnSOD-positive structures were found in the bronchiolar epithelium of the lungs obtained at each time point. Again, the positive structures increased with age (Fig. 3). There was a significant staining for MnSOD in the neonatal alveolar wall including the epithelium, capillary endothelium, and mesothelium (Fig. 4), but those structures in the fetal lung were not stained for MnSOD. The age-related increase in the staining for each SOD in the lung was clearly demonstrated by morphometric measurement (Table 2). The area percentage of the staining for $\mathrm{CuZnSOD}$ was 3-fold higher in the neonatal lung than in the lung of the $\mathrm{d} 18$ fetus. That for MnSOD increased more than 20 -fold during this period.

In the fetal and neonatal kidneys, well-developed glomeruli were found mainly in the juxtamedullary region of the cortex. The tubular epithelium was stained for both SOD, but there was no staining in the glomeruli and interstitial space. The renal tubules had not yet been differentiated into proximal and distal types, even in the neonatal kidneys. They were all stained moderately, with equal intensity, for both CuZnSOD and MnSOD (Figs. 5 and 6). As reflected in the morphometric data (Table 2), tubular mass increased rapidly during this period. The area percentage of the staining for CuZnSOD increased almost 3-fold, and that for MnSOD increased approximately 5 -fold.

\section{DISCUSSION}

There have been studies on the immunolocalization of $\mathrm{Cu}$ $\mathrm{ZnSOD}$ in adult rat lungs $(8,10,11)$. According to these studies, $\mathrm{CuZnSOD}$ reactivity was intense in bronchiolar epithelium and moderate in alveolar wall. Thus, the relative abundance of CuZnSOD in the bronchiolar epithelium of both the fetal and neonatal lungs observed here was similar to the distribution in the adult lungs. The finding that CuZnSOD staining was more intense in the neonatal lung than in the fetal lung was consistent with the increase in the immunoreactive SOD in the lung homogenate during this period.

In our previous study (8), both bronchiolar epithelium and alveolar wall in adult rat were stained for MnSOD moderately, with equal intensity. The lower MnSOD reactivity in the neonatal alveolar wall than in the bronchiole and no reactivity in the fetal alveolar wall observed here indicate that MnSOD accumulates in the alveolar wall later than in the bronchiolar epithelium. The finding that the intensity of the staining increased during late gestation in the whole lung tissues was, again, in keeping with the observed drastic increase in MnSOD measured by the RIA.

A rapid structural development is known to occur in lungs during late gestation: branching and lengthening of terminal tubules, marked widening of the air spaces resulting in the formation of acini with air-blood barriers, and differentiation of alveolar epithelial cells into several types (12). Cellular differentiation may contribute to the increase in both SOD (13). Lung CuZnSOD (14) is reported to be induced by exposure to high concentrations of oxygen. We previously demonstrated that lung SOD increased during late gestation without exposure to oxygen (7). Hass et al. (15) measured mRNA in developing rat lung. They suggested that there could be both pretranslational and translational regulation in the induction of CuZnSOD by oxygen exposure. However, the cellular mechanism for the induction still needs to be elucidated.

Hyperoxia increases superoxide production in rat lung mitochondria (16), and is postulated to be a major cause of bronchopulmonary dysplasia (17). Induction of endogenous antioxidant enzymes by the administration of bacterial lipopolysaccharide is reported to protect against oxygen lung injury (18). The lung injury is supposed to be initiated by microvascular endothelial damage (19), leading to the destruction of the alveolar wall. This can be ameliorated by i.v. injection of liposome-entrapped catalase and SOD (20). The low concentration of both SOD in the fetal alveolar wall including capillary endothelium, which is assumed to be particularly vulnerable to oxidant attack, further supports our previous view (7) that preterm lung is vulnerable to oxygen toxicity because of the scarcity of the antioxidant enzymes.

According to previous studies $(8,10)$ on adult rat kidneys, CuZnSOD reactivity was most intense in proximal tubules, less intense in the rest of the tubules and ducts, and not found in glomeruli. On the other hand, Henle's loops and collecting ducts were more intensely stained for MnSOD than either proximal or distal tubules, and glomeruli were not stained (8). In general, cortex was rich in CuZnSOD and, conversely, medulla was rich in MnSOD (8). It is known that rat kidneys are immature at birth. There is an active nephrogenic zone in which new glomeruli and tubules are still undergoing the phases of development (21). Lack of the differential distribution found in the adult kidney for both SOD was due to the immaturity of the neonatal kidney. However, the finding that the stainings for both SOD were confined to the undifferentiated tubules in the fetal and neonatal kidneys was consistent with the staining profile in adult rat kidneys in which the reactivities were restricted to the tubules and ducts (8)

The observed drastic increase in the immunoreactivities in the kidney homogenates is, most likely, mainly due to the rapid proliferation of the tubular epithelium during this period. There is evidence suggesting that both glomerular and tubular damage can be mediated by reactive oxygen species $(22,23)$. Tubules are vulnerable to reactive oxygen species, even in the presence of a high concentration of antioxidant enzymes, presumably because the rate of production of reactive oxygen species is high in these 
metabolically active sites. Whether or not preterm kidneys are vulnerable to oxidative stress is unknown. In contrast to our present observations that the expression of both SOD in specific tissues was intensified in the lungs during late gestation, the intensity of the staining in the renal tubules was unchanged. This may be related to the finding that the tubules were still undifferentiated even in the neonatal kidneys. Thus, the low levels of both SOD in fetal kidneys may merely reflect the scarcity of tubular mass rather than vulnerability to oxidative stress.

Our present histochemical study disclosed that both $\mathrm{CuZnSOD}$ and MnSOD increased in the kidneys in a different manner than they did in the lungs, and this difference could not be depicted by the biochemical study.

Acknowledgment. The authors gratefully acknowledge the technical assistance of Hiromi Horiuchi.

\section{REFERENCES}

1. Yam J, Frank L, Roberts RJ 1978 Age-related development of pulmonary antioxidant enzymes in the rat. Proc Soc Exp Biol Med 157:293-296

2. Yoshioka T, Shimada T, Sekiba K 1980 Lipid peroxidation and antioxidants in the rat lung during development. Biol Neonate 38:161-168

3. Tanswell AK. Freeman BA 1984 Pulmonary antioxidant enzyme maturation in the fetal rat. I. Developmental profiles. Pediatr Res 18:584-587

4. Gerdin E, Tyden O, Eriksson UJ 1985 The development of antioxidan enzymatic defense in the perinatal rat lung: activities of superoxide dismutase, glutathione peroxidase, and catalase. Pediatr Res 19:687-691

5. McCord JM 1988 Free radicals and myocardial ischemia: overview and out look. Free Radic Biol Med 4:9-14

6. Asayama K, Burr IM 1985 Rat superoxide dismutases: purification labeling, immunoassay and tissue concentration. J Biol Chem 260:2212-2217

7. Hayashibe H, Asayama K, Dobashi K, Kato K 1990 Prenatal development of antioxidant enzymes in rat lung, kidney, and heart: marked increase in immunoreactive superoxide dismutases, glutathione peroxidase, and catalase in the kidney. Pediatr Res 27:472-475
8. Dobashi K Asayama K Kato K, Kobayashi M, Kawaoi A 1989 Immunohistochemical localization of copper-zinc and manganese superoxide dismutases in rat tissues. Acta Histochem Cytochem 22:351-365

9. Beier F, Fahimi HD 1986 Application of automatic image analysis for morphometric studies of peroxisomes stained cytochemically for catalase. Cell Tissue Res 246:635-640

10. Thaete LG, Crouch RK, Spicer SS 1985 Immunolocalization of copper-zinc superoxide dismutase. II. Rat. J Histochem Cytochem 33:803-808

11. Mossman BT, Marsh JP, Shatos MA 1986 Alteration of superoxide dismutase activity in tracheal epithelial cells by asbestos and inhibition of cytotoxicity by antioxidants. Lab Invest 54:204-212

12. Ballard PL 1986 Lung development. In: Hormone and Lung Maturation Springer-Verlag, New York, pp 1-23

13. Allen RG, Balin AK 1989 Oxidative influence on development and differentiation: an overview of a free radical theory of development. Free Radic Biol Med 6:631-661

14. Crapo JD, McCord JM 1976 Oxygen-induced changes in pulmonary superoxide dismutase assayed by antibody titrations. Am J Physiol 231:11961203

15. Hass MA, Iqbal J, Clerch LB, Frank L, Massaro D 1989 Rat lung Cu.Zn superoxide dismutase: isolation and sequence of a full-length $\mathrm{CDNA}$ and studies of enzyme induction. J Clin Invest 83:1241-1246

16. Freeman BA, Crapo JD 1981 Hyperoxia increases oxygen radical production in rat lungs and lung mitochondria. J Biol Chem 256:10986-10992

17. Frank L, Sosenko IRS 1987 Development of lung antioxidant enzyme system in late gestation: possible implications for the prematurely born infant. $J$ Pediatr 110:9-14

18. Frank L Summerville J, Massaro D 1980 Protection from oxygen toxicity with endotoxin: role of the endogenous antioxidant enzymes of the lung. J Clin Invest 65:1104-1110

19. Brigham KL 1986 Role of free radicals in lung injury. Chest $89: 859-863$

20. Turrens JF, Crapo JD, Freeman BA 1984 Protection against oxygen toxicity by intravenous injection of liposome-entrapped catalase and superoxide dismutase. J Clin Invest 73:87-95

21. Speller AM, Moffat DB 1977 Tubulo-vascular relationships in the developing kidney. J Anat 123:487-500

22. Shah SV 1989 Role of reactive oxygen metabolites in experimental glomerula disease. Kidney Int 35:1093-1106

23. Paller MS, Hoidal JR, Ferris TF 1984 Oxygen free radicals in ischemic acute renal failure in the rat. $J$ Clin Invest 74:1156-1164 